\title{
Analysis and Characterization of Embedded Vision Systems for Taxonomy Formulation
}

\author{
Muhammad Imran*a, Khaled Benkrid ${ }^{\mathrm{b}}$, Khursheed Khursheed ${ }^{\mathrm{a}}$, Naeem Ahmad ${ }^{\mathrm{a}}$, Mattias O'Nils ${ }^{\mathrm{a}}$, \\ Najeem Lawal ${ }^{\text {a }}$, \\ ${ }^{a}$ Department of Information Technology and Media, Mid Sweden University, Sweden, \\ ${ }^{\mathrm{b}}$ School of Engineering at the University of Edinburgh, Scotland, UK.
}

\begin{abstract}
The current trend in embedded vision systems is to propose bespoke solutions for specific problems as each application has different requirement and constraints. There is no widely used model or benchmark which aims to facilitate generic solutions in embedded vision systems. Providing such model is a challenging task due to the wide number of use cases, environmental factors, and available technologies. However, common characteristics can be identified to propose an abstract model. Indeed, the majority of vision applications focus on the detection, analysis and recognition of objects. These tasks can be reduced to vision functions which can be used to characterize the vision systems. In this paper, we present the results of a thorough analysis of a large number of different types of vision systems. This analysis led us to the development of a system's taxonomy, in which a number of vision functions as well as their combination characterize embedded vision systems. To illustrate the use of this taxonomy, we have tested it against a real vision system that detects magnetic particles in a flowing liquid to predict and avoid critical machinery failure. The proposed taxonomy is evaluated by using a quantitative parameter which shows that it covers 95 percent of the investigated vision systems and its flow is ordered for 60 percent systems. This taxonomy will serve as a tool for classification and comparison of systems and will enable the researchers to propose generic and efficient solutions for same class of systems.
\end{abstract}

Keywords: system taxonomy, embedded vision systems, smart cameras, wireless vision sensor nodes.

\section{INTRODUCTION}

Vision systems can be implemented either on conventional, relatively higher performance computer systems or on embedded ones. Conventional computer systems often have little limitation on resources e.g. memory, and as a result, there is a large degree of flexibility in their design and implementation. However, such systems are difficult to deploy for many embedded applications, often suffer from high latency and throughput limitations, and require relatively high installation and maintenance cost. In comparison, embedded vision systems are comparatively lower cost, lower power, more tuned towards real time performance, and easier to install and maintain. Therefore, developing vision systems for embedded applications requires different design and implementation considerations and challenges. Overall, the field of embedded vision systems is still immature and there is a need to systematize the design of such systems, at least for benchmarking and evaluation purposes. There exist many different ways to propose solutions for a particular vision system problem. Mostly, the emphasis is first narrowed down to particular solutions out of many alternatives. It is hard to generalize the flow of vision processing for different applications as each vision problem has different requirements. Many vision functions are however common in embedded vision systems, developed for a wide range of applications. Indeed, the majority of vision applications target detection, analysis and recognition of objects present in the field of view $^{1}$. This provides motivation that common characteristics of embedded vision systems can be identified, with vision functions being considered as a main differentiating parameter.

The aim of this paper is to present a vision system taxonomy that can be used as a reference model for classification and comparison of different embedded vision systems. When embedded vision systems are classified by using the taxonomy, generic and efficient solutions to different classes can then be developed. We showed in this work how a particular system can be classified according to the proposed taxonomy. In order to show how well the taxonomy covers the existing systems, we have tested it with number of real vision systems in a quantitative way. Although we have analyzed many different vision systems, wired and wireless, individual standalone vision systems and wireless smart camera in

*muhammad.imran@miun.se; phone +46 (0) 601484 16; fax +46 (0) 601484 56; miun.se 
Wireless Vision Sensor Networks (WVSNs), for problem formulation, our focus is on embedded vision system particularly wireless smart camera used in Wireless Vision Sensor Network (WVSNs). The remainder of this paper is organized as follows. Section 2 first presents related work and section 3 describes the problem. Section 4 then covers the vision characteristics of embedded vision systems. After that, Section 5 presents our proposed system taxonomy. Then, section 6 discusses the results. Finally, section 7 summarizes the conclusions drawn from this work.

\section{RELATED WORK}

Before elicitation of the problem to be solved, this section will first present a sample of work published in the literature in the area of embedded visions systems. Rinner et $a l .^{2}$ classify smart camera research based on platform capabilities, the degree of distributed processing as well as system autonomy. Some of the existing vision systems implemented on smart cameras are described and classified according to the proposed taxonomy. In addition, some of the challenges associated with smart camera systems are described. Hengstler et al. ${ }^{33}$ proposed an application oriented design methodology for a smart camera. However, the authors consider only the specific case of tracking objects using a single camera and a stereo vision smart camera network. Dieber et $a l .^{32}$ the authors presented a formulation and approximation method for the camera selection and task assignment in order to achieve the required monitoring activities. The trade-off between surveillance quality and resource utilization has been investigated. Tilak et $a l^{34}$ classified the wireless micro-sensor networks from a communication protocol perspective. Different types of communication functions, data delivery models and network dynamics are discussed for wireless sensor networks; however, there is no discussion in relation to camera based sensor systems. Generally, the camera based sensors produce two dimensional data which puts strick requirements on processing capabilities, memory, power consumption and communication bandwidth. Kulkarni et al. ${ }^{4}$ proposed a multi-tier architecture, called SensEye. In this, focus is on three surveillance tasks namely, object detection, recognition and tracking. The Sensor node ${ }^{5}$ captures the raw data, objects are detected and then objects of interest are forwarded. The cameras node can collaborate and transmit the textual description along with a snapshot.

An investigation of the aforementioned systems shows that the focus is on particular aspects of the vision systems. There is no common mechanism for classification and comparison of different vision systems, which is necessary for improving the research in this field. In order to fill this gap, we have proposed a system taxonomy which covers the functionality of different class of wireless smart camera systems. To the best of our knowledge, this will be first contribution which provides reference model in the form of system taxonomy, for vision systems implemented on embedded platforms.

\section{PROBLEM ELICITATION}

The plethora of varied visions systems reported in the literature makes it necessary to provide a benchmark or at least an abstract model of embedded vision systems which can be used as a reference tool. In order to develop this, it is necessary to thoroughly analyze existing vision systems and identify the problem space. We have indeed surveyed a large number of vision systems and found that that there is an opportunity to develop an abstract model for embedded vision system classification. In order to achieve this, we aim to develop a system taxonomy which is based on the vision functions and their combination. The mechanism we adopted to develop this taxonomy is shown in Figure 1 in which the problem space is to identify common vision characteristics of different embedded vision systems. By looking at a large number of vision systems, it is concluded that a relatively limited number of vision functions can be used to characterize embedded vision systems. Similar vision functions are grouped together to make an abstraction of vision functions. These abstracted vision functions are then used to define the system taxonomy.

Taxonomy building is however an iterative process represented back and forth arrows in Figure 1, therefore, we have to look into emerging systems continuously in order to ensure appropriate coverage of different types of embedded vision systems. By looking into existing systems and comparing them with system taxonomy, improvements can be identified and relevant changes can be made on abstraction level and system taxonomy. In this work, we have adopted this iterative approach for the development of system taxonomy. The taxonomy shows system functionality rather than implementation and complexity.

\section{CHARACTERISTICS OF EMBEDDED VISION SYSTEMS}

From our in-depth analysis of published literature for vision systems, we have identified the following characteristics of embedded vision systems: 


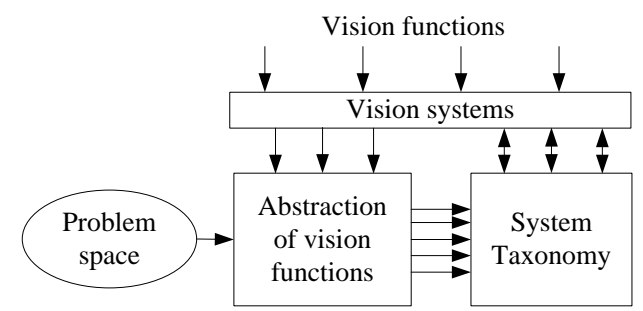

Figure 1. Problem flow and taxonomy building process

\subsection{Data Trigger, acquisition and width}

Vision systems can be divided into three categories based on how the system is triggered to start the vision processing, namely: time driven, event/query driven and period driven.

Vision systems acquire images through cameras which are categorized as either area array or line scan array. In some applications, sensor nodes exchange information with each other for some specific applications to improve performance and reliability.

Depending on the application, camera sensors with different bits per pixel can be employed for image capturing as the conversion from one image format to another would require additional resources ${ }^{11}$. Images can be captured using various formats such as binary, grey or in colour.

\subsection{Colour Transformation}

Colour transforms involve conversion of colour information from one colour space to another, conversion from colour to grey scale. In some applications, it is required to convert the image from RGB to the HSI or YUV colour space. For example, colour segmentation works better in HSI and YUV ${ }^{36}$.

\subsection{Learning of the system}

For some applications, the system needs to be trained so that it is adapted to the environment. This trend will enable the vision systems with more complex forms of awareness about the view being captured or context of the events ${ }^{18}$.

\subsection{Frame/background storage and subtraction}

Depending on the applications, embedded vision systems can have three types of storage requirements: namely No storage ${ }^{35}$, initial storage ${ }^{19}$ or continuous storage ${ }^{5}$.

The common method in image processing to highlight useful information is to perform background subtraction in which background is subtracted from foreground. For background subtraction, the background image may either be stored in memory initially ${ }^{19}$ or generated in real time using background modelling techniques ${ }^{16}$.

\subsection{Segmentation and filtering}

Image segmentation is the process of spatial partitioning of an image into mutually exclusive connected image regions or objects. Segmentation techniques include: manual thresholding, adaptive thresholding, edge based, region based, segmentation using morphological watersheds ${ }^{17}$.

Image filtering approaches fall into two broad categories: linear digital filtering and nonlinear digital filtering, based on the computation performed on neighbour pixels. Taking into account the noise behaviour, filtering can be performed in the frequency or spatial domain ${ }^{17}$

\subsection{Labelling and feature extraction}

Different labelling algorithms include component labelling using single pass, two passes, multiple scan algorithms, parallel algorithms, contour tracing techniques algorithm ${ }^{21}$.

Some applications require that quantitative parameters of objects are extracted from regions or contours. These quantitative parameters show the characteristics of the objects and are usually called features. Different types of features include region features, grey value features and contour features ${ }^{22}$. 


\subsection{Classification, recognition and tracking}

Classification is mapping from feature space to set of possible classes. When a feature set and a training set of image data is given, any of the machines learning approach can be selected for classification function ${ }^{22}$.

Pattern recognition is a process which combines the aforementioned algorithms to detect a single or multiple objects in the image. Recognition can be achieved by using template matching. Template matching approaches are based on finding similarity between template and image using the grey values of the template and the image or by using the closeness of the template edges to the image edges ${ }^{22}$.

Tracking involves continuous object detection which can be achieved by using object recognition. Object tracking, in general is a challenging problem due to abrupt object motion, changing appearance patterns of object and scene, object to object and object to scene occlusions and camera motion ${ }^{23}$.

\subsection{Transformations}

Transformation includes intensity transformation, spatial transformation and mathematical transformation.

Intensity transformation is a spatial domain category of image processing operations, working on single pixels of an image. Different intensity transforms used for image enhancement include linear, logarithmic, power law, piecewiselinear transformation and histogram processing ${ }^{17}$.

Spatial transforms are pixel level mapping and describe operations that change the position of the data. These included image transform, matrix transpose, image rotate, image scaling and image registration ${ }^{24}$.

The mathematical transform includes discrete cosine transforms (DCTs), fourier transforms, wavelet, and hough transforms ${ }^{24}$. In some cases, it is better to perform operations on image, transformed in another domain. The choice of a particular transform in a given application depends on the amount of reconstruction error that can be tolerated and the computational resources available ${ }^{17}$.

\subsection{Post processing}

Post processing includes compression and other functions which are used for formatting the data in order to make it suitable for communication. The compression schemes are broadly categorized in two types, namely lossless compression and lossy compression.

\subsection{Output data type and feedback}

The amount of data being transmitted from the embedded vision systems has a great impact on the energy consumption in case of WVSNs ${ }^{19}$. As a result, there is a need to investigate the type of data that needs to be transmitted. The output of the WVSN can be in different formats such as in matrix format, scalar, vector or flag depending on the application.

The advancement in image processing equipment enabled the use of vision as feedback signal ${ }^{25}$. High speed realization of visual feedback requires that image processing is performed as fast as the feedback rate ${ }^{26}$. Feedback for the system can be sent from different stages of processing. The feedback can be control signals in the form of a matrix, vector, scalar or flag.

Following these characteristics, system taxonomy will be presented for embedded vision systems.

\section{PROPOSED SYSTEM TAXONOMY}

Our proposed vision system taxonomy is developed according to the flow shown in Figure 1. Vision functions and their combination are used to characterize the taxonomy. Similar functions were grouped together to make an abstraction of vision functions. The abstracted vision functions are then used to develop our proposed vision system taxonomy as shown in Figure 2. Some of the functions are labelled by capital letters in Figure 2, which are further expanded from Figure 3 to Figure 9.

The system taxonomy is grouped into 9 levels including data triggering type, data acquisition, data width, learning, storage requirement, vision processing, post processing, output type and feedback. An embedded vision system is categorized into three types based on how the system is triggered to start processing. In time driven systems, the processing is performed after certain time duration. In event/query driven systems, processing starts when a system is triggered by certain event or query. Periodic systems start processing after a fixed duration of time. The system can 


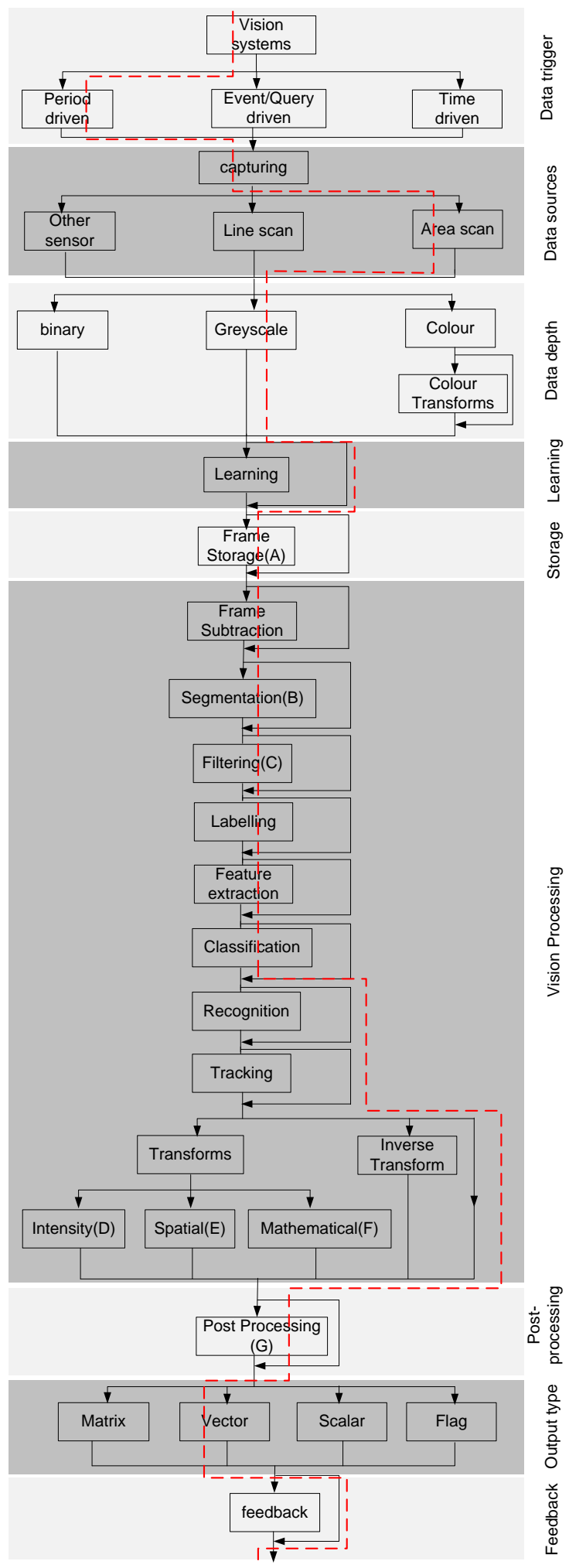

Figure 2. Taxonomy of vision system. The associated functions with labels are shown in Figure 3 to Figure 8 .

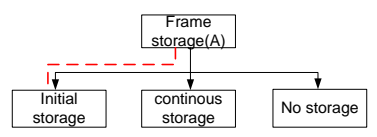

Figure 3. Storage in vision systems

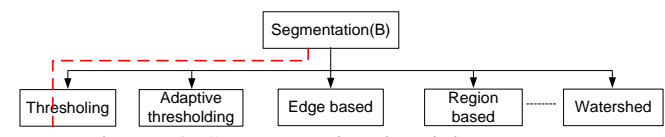

Figure 4. Segmentation in vision systems

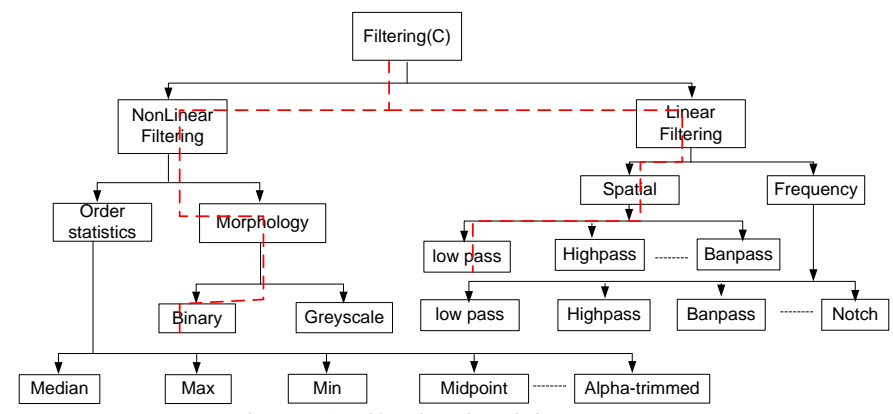

Figure 5. Filtering in vision systems

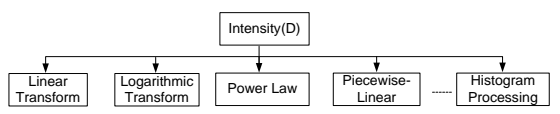

Figure 6. Intensity Transformation in vision systems

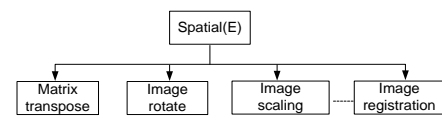

Figure 7. Spatial Transformation in vision systems

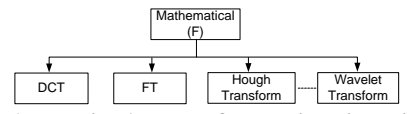

Figure 8. Mathematical Transformation in vision systems

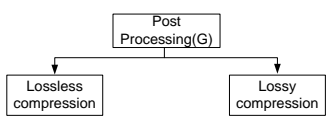

Figure 9. Post processing

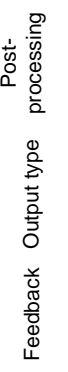


receive data using three types of sources including: area scan, line scan or from another system. Images can be captured in binary, grey scale or in colour format. Conversion from one format to another would require additional resources. Therefore, the best strategy is to capture the image in the relevant format, as discussed in sub-section 4 . For some applications, the systems learn about the environment to adapt to it while in some applications there is no need for learning. Similarly, there could be requirement for storage in some systems in order to store frames while in others, there is no need of storage. The vision processing level in Figure 2 shows the abstraction of vision functions which while not exhaustive includes typical processing functions required for embedded vision systems. After the processing level, post processing occurs. Note that in general, functions have an alternative path to circumvent them to show that they might not be required for some systems. An embedded vision system is also characterized by the output type, it produces. The output can be a matrix, vector, scalar or flag and can be sent directly to the user or can be used for feedback. In some applications, feedback can produce better results as compared to system without feedback ${ }^{16}$. It must be noted that the system taxonomy does not show complexity or the vision flow of the systems because systems can take on different function order to achieve same results. After presenting the system taxonomy, one published system ${ }^{19}$ is described in more detail in relation to the taxonomy. The system is mapped on the proposed taxonomy and individual vision functions are discussed.

\subsection{Case Study}

To illustrate the use of our taxonomy, this section presents a WVSN system ${ }^{19}$ which is developed to detect magnetic particles in flowing liquid, measure their sizes and transmit the features' information to an external agent. Such automatic detection is crucial for failure detection in machinery. Note that flowing liquid in the system might contain air bubbles which can be identified as objects. The removal of such bubbles is hence necessary. The vision function flow is shown in Figure 10.

In this system, image capturing is performed by using CMOS area scan image sensor (MT9V032) from Micron ${ }^{27}$. In background subtraction current frame is subtracted from initially stored background in flash memory. Following this manual thresholding is applied for segmentation as the objects that need to be detected are white and the background is relatively black i.e. there is a large degree of contrast. In the morphology operation erosion is applied, followed by dilation with a mask of $3 \times 3$. In bubble removal which is basically a spatial domain low pass filtering stage, unwanted objects are removed.

During labelling, pixels belonging to the same image component are assigned a unique label. A single pass connected component labeling was used for this purpose in order to achieve real time component labelling ${ }^{19}$. At feature extraction, an image component is described in terms of region features such as area, mean grey value or position. This feature information can then be used for the classification of image components. The output data is in the form of vector containing objects/particles areas, number of particles and position information. This vector is transmitted to an external agent using IEEE 802.15.4 compliant transceiver.

The system is classified by using our proposed taxonomy as shown in Figure 2 where the dashed line represents the case study system's flow. The expanded functions are labelled by capital letter such as storage by A, segmentation by B, etc. These functions are shown from Figure 3 to Figure 5, with similar dashed lines. In this system, processing is performed periodically (i.e. it is a period driven wireless smart camera) and an area scan camera is used for capturing images. Background is stored initially when there are no objects in the field of view. Other vision functions include: background subtraction, greyscale segmentation, filtering, labelling, feature extraction and classification. The output data is in the form of vector. The system classification with respect to the proposed taxonomy shows that all functions of the system are covered and ordered for this system. In a similar way, other systems can be classified and generalized solutions can be proposed for same class of systems. The following section will provide an evaluation of our proposed taxonomy.

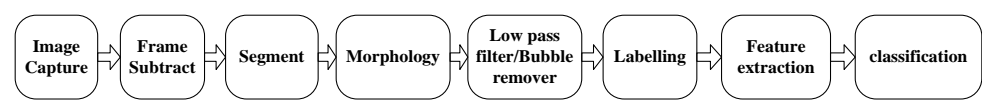

Figure 10. Algorithm flow of different processing strategies

\subsection{Evaluation mechanism of Taxonomy}

Taxonomy evaluation is a challenging task due to large number of influencing factors, many of which are difficult to quantify. The outcome of such evaluation is mostly a subjective judgment. However, it is necessary to conduct an 


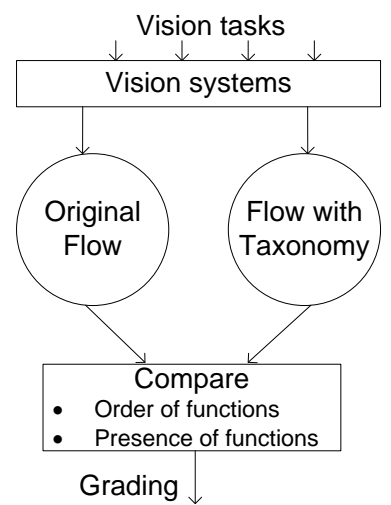

(a)

Presence of Functions Grading

$\mathrm{I}=$ (No function missing)

II $=(1-2$ functions missing $)$

III $=(3-4$ functions missing $)$

$\mathrm{IV}=(>4$ functions missing $)$

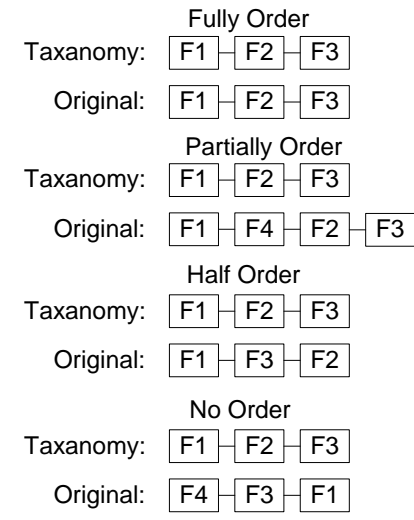

(b)

Order of functions

$A=$ Fully order

$\mathrm{B}=$ Partially order

$\mathrm{C}=$ Half Order

$\mathrm{D}=$ No Order

Figure 11. Evaluation criteria of Taxonomy

educated discussion about this judgment. Our objective is to get quantitative parameters to grade our proposed taxonomy in relation to different vision systems. These parameters will show how suitable is the taxonomy and how well it covers existing vision systems. The evaluation mechanism is shown in Figure 11. The vision system's flow is compared with the taxonomy flow. Two parameters are used for this purpose, namely the presence of the taxonomy functions and their order. These two parameters are graded in four levels, with I, II, III, IV used for function presence, and A, B, C and D used for function order.

\section{RESULT AND DISCUSSION}

The vision systems used for taxonomy evaluation are shown in Table 2. The flow of the vision functions based on the taxonomy is shown in column2, whereas the system's actual flow is shown in column3. The first evaluation criterion is: how well the taxonomy covers the system's functions? We have allotted four grades to this criterion. The taxonomy is graded I when there is no missing functions in the taxonomy compared to the vision system under study. Grade II is assigned when one to two functions are missing, III is assigned when three or four functions are missing, and finally grade IV is assigned when more than four functions are missing in the taxonomy. Another parameter to evaluate is the ordering of functions in the taxonomy in comparison to the system's actual flow. We have allotted four levels to this criterion including fully ordered (grade A), partially ordered (grade B), half ordered (grade C) and not ordered (grade D). This is shown in Figure 11 (b).

The taxonomy is fully ordered when the flow of the vision functions for any vision system is similar to the flow of the functions derived from the taxonomy. The taxonomy is partially ordered when the vision system under study follows the taxonomy flow, except for some additional functions in between the ordered functions of the investigated system. Half ordered grade is assigned when taxonomy can be fully or partially ordered by shifting one or two vision functions of taxonomy to bring order similar to the investigated system. Finally, a no ordering grade is allocated when more than two functions are required to bring half/full order. These gradings are shown in column 4 and column5 of Table 2. For instance, in the first row in Table 2, the presence of functions has I grade as all functions of this system are present in taxonomy. Similarly, vision system is fully ordered and has hence a grade A for ordering. For the second system, two functions, downsampling and centroid to grammer, are missing in the taxonomy.

This shows that some functions will inevitably be missing in the taxonomy for certain vision systems because of specific requirements. The absence of two functions in system 2 results in grade II for functions presence. The presence of down sampling in between the ordered functions results in B grade. An evaluation summary is shown in Table 1 in which it is evident that the taxonomy's vision functions provide a good coverage of the vision systems reported in the literature. The system taxonomy has highest grade I for 55 percent systems and grade II for 40 percent systems (i.e. for function 
Table 1. Comparison results of 20 systems

\begin{tabular}{|l|l|l|l|l|l|}
\hline \multicolumn{2}{|l|}{ Functions presence } & \multicolumn{3}{l|}{ Functions order } \\
\hline Grade & frequency & \%age & Grade & frequency & \%age \\
\hline I & 11 & 55 & A & 7 & 35 \\
\hline II & 8 & 40 & B & 5 & 25 \\
\hline III & 1 & 5 & C & 7 & 35 \\
\hline IV & 0 & 0 & D & 1 & 5 \\
\hline
\end{tabular}

presence) out of the total investigated systems. It means that the taxonomy covers 95 percent of the investigated systems in which either no function is missing or at most 2 functions are missing in taxonomy.

Regarding ordering, out of the total investigated systems, the taxonomy has A grade (fully ordered) for 35 percent systems, B grade (partially ordered) for 25 percent systems, C grade (half ordered) for 35 percent systems and D grade (no order) for 5 percent system. This shows that there is more variability in terms of ordering than the presence of functions. Overall, the function presence criterion gives good support for our proposed taxonomy. The missing functions for some systems are problem specific as in case of system 5, where feature fusion is required. In general, missing vision functions of taxonomy are shown in italic font in cloumn 3 of Table 2 . Note that some systems' functionality can be achieved by using existing taxonomy functions. These functions are considered as present in Table 2 e.g. background modelling in systems, identified by $*$, such as system 11, 12 and 15, can be achieved by using filtering techniques or background can be stored at the initial stage when there is no object in the field of view. With regards to ordering, Table 1 shows that our system samples have A and B grade for 60 percent of the systems and C and D grade for the remaining 40 percent.

The system taxonomy indeed is not intended to show the exact flow of the functions rather it shows the functionality of the vision systems. For example, filtering is a function which may be implemented before segmentation ${ }^{28}$ or after segmentation ${ }^{19}$, depending on the requirement of the application, and this variability will always be present depending on the application. Nonetheless, it is useful to provide taxonomy in the form of an abstract model of embedded vision systems against which benchmarks can be obtained, and generic and efficient architectures for different grades or classes of embedded vision systems can be developed. It will lead to better comparisons and evaluations, as well as reduced design and development time of vision systems implemented on embedded platforms.

\section{CONCLUSION}

In this paper, we presented a system taxonomy with the aim of providing an abstract model of vision systems which will facilitate the benchmarking and development of generic and efficient solutions for vision systems implemented on embedded platforms. Central to our proposed system taxonomy is a limited number of vision functions suffice to cover a large number of real visions systems reported in the literature.

We have tested 20 visions systems from the literature against our proposed taxonomy in order to show the maturity of our taxonomy. The evaluation criterion for the proposed system taxonomy was based on two parameters: 1) functions presence, and 2) the ordering of functions in the taxonomy in relation to the actual vision system ordering. This showed that the taxonomy covers 95 percent of the investigated systems in terms of function presence. Function ordering however showed more variability, as the taxonomy was fully/partially ordered for 60 percent of the investigated systems. Indeed, our system taxonomy focuses on the functionality of vision systems rather than the exact ordering of functions which depends on the details of the application at hand. To illustrate the use of our proposed taxonomy, we have tested it against a real vision system that we have developed to automatically detect magnetic particles in flowing liquid to predict and avoid critical machinery failure.

We hope that our proposed taxonomy will provide a realistic model of vision system implemented on embedded platforms which can be developed in the future by the community at large. This will facilitate fairer benchmarking and evaluation of embedded vision systems, as well as the development of generic and efficient solutions for different grades or classes of systems. 
Table 2. Systems with Taxonomy flow vs Actual flow

\begin{tabular}{|c|c|c|c|c|}
\hline \multirow{2}{*}{ 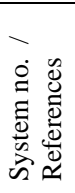 } & \multirow[t]{2}{*}{ System flow derived from taxonomy } & \multirow[t]{2}{*}{ System flow in published work } & \multicolumn{2}{|c|}{ Functions } \\
\hline & & & 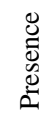 & $\frac{\bar{s}}{0}$ \\
\hline $1^{19}$ & $\begin{array}{l}\text { Tasks: capture, frame storage, subtract, segment, filtering, } \\
\text { labelling, feature extraction, classification. } \\
\text { Output type: vector }\end{array}$ & $\begin{array}{l}\text { Tasks: capture, frame storage, subtract, segment, filtering, } \\
\text { labelling, feature extraction, classification. } \\
\text { Output type: vector }\end{array}$ & I & A \\
\hline $2^{3}$ & $\begin{array}{l}\text { Tasks: capture, training, frame storage, subtract, filtering. } \\
\text { Output type: scalar }\end{array}$ & $\begin{array}{l}\text { Tasks: capture, downsampling, training, frame storage, } \\
\text { frame difference, edge detect, centroid calculation, centroid } \\
\text { grammer. } \\
\text { Output type: scalar }\end{array}$ & II & B \\
\hline $3^{12}$ & $\begin{array}{l}\text { Tasks: capture, training, frame storage, subtract, feature } \\
\text { extraction, classification. } \\
\text { Output type: vector }\end{array}$ & $\begin{array}{l}\text { Tasks: capture, training, frame storage, subtract, } R O I \text {, } \\
\text { feature extraction, classification. } \\
\text { Output type: vector }\end{array}$ & II & B \\
\hline $4^{26}$ & $\begin{array}{l}\text { Tasks: capture, colour transform, frame storage, } \\
\text { mathematical transform. } \\
\text { Output type: vector }\end{array}$ & $\begin{array}{l}\text { Tasks: capture, colour transform, frame storage, DCT. } \\
\text { Output type: vector }\end{array}$ & I & A \\
\hline $5^{28}$ & $\begin{array}{l}\text { Tasks: capture, training, subtract, segment, filtering, } \\
\text { intensity transform, labelling, feature extraction. } \\
\text { Output type: vector }\end{array}$ & $\begin{array}{l}\text { Tasks: capture, training, subtract, median-erode-dilate, } \\
\text { segment, labelling, histogram, feature extraction, feature } \\
\text { fusion. } \\
\text { Output type: vector }\end{array}$ & II & $\mathrm{C}$ \\
\hline $6^{6}$ & $\begin{array}{l}\text { Tasks: capture, frame storage, subtract, segment, feature } \\
\text { extraction. } \\
\text { Output type: vector }\end{array}$ & $\begin{array}{l}\text { Tasks: capture, frame storage, subtract, segment, ROI, } \\
\text { features of ROI. } \\
\text { Output type: vector }\end{array}$ & II & B \\
\hline $7^{30}$ & $\begin{array}{l}\text { Tasks: capture, frame storage, subtract, segment, filtering, } \\
\text { feature extraction, tracking. } \\
\text { Output type: vector }\end{array}$ & $\begin{array}{l}\text { Tasks: capture, frame storage, subtract, median, segment, } \\
\text { bounding box, tracking. } \\
\text { Output type: vector }\end{array}$ & I & $\mathrm{C}$ \\
\hline $8^{29}$ & $\begin{array}{l}\text { Tasks: capture, frame storage, subtract, segment, intensity } \\
\text { transform. } \\
\text { Output type: vector }\end{array}$ & $\begin{array}{l}\text { Tasks: capture, frame storage, subtract, histogram, } \\
\text { segment. } \\
\text { Output type: vector }\end{array}$ & I & $\mathrm{C}$ \\
\hline $9^{11}$ & $\begin{array}{l}\text { Tasks: capture, training, frame storage, subtract, segment, } \\
\text { labelling, classification. } \\
\text { Output type: vector }\end{array}$ & $\begin{array}{l}\text { Tasks: capture, training, frame storage, subtract, segment, } \\
\text { labelling, classification. } \\
\text { Output type: vector }\end{array}$ & I & A \\
\hline $10^{5}$ & $\begin{array}{l}\text { Tasks: capture, frame storage, subtract, segment, filtering, } \\
\text { feature extraction. } \\
\text { Output type: vector }\end{array}$ & $\begin{array}{l}\text { Tasks: capture, frame storage, subtract, segment, low pass } \\
\text { filtering, bounding box. } \\
\text { Output type: vector }\end{array}$ & I & A \\
\hline $11 * 16$ & $\begin{array}{l}\text { Tasks: capture, frame storage, subtract, segment, feature } \\
\text { extraction, tracking, feedback. } \\
\text { Output type: vector }\end{array}$ & $\begin{array}{l}\text { Tasks: capture, frame storage, subtract, segment, feature } \\
\text { extraction, tracking, feedback. } \\
\text { Output type: vector }\end{array}$ & $\mathrm{I}$ & A \\
\hline $12^{* 8}$ & $\begin{array}{l}\text { Tasks: capture, frame storage, subtract, mathematical } \\
\text { transform. } \\
\text { Output type: vector }\end{array}$ & $\begin{array}{l}\text { Tasks: capture, frame storage, subtract, mathematical } \\
\text { transform. } \\
\text { Output type: vector }\end{array}$ & I & A \\
\hline $13^{9}$ & $\begin{array}{l}\text { Tasks: capture, segment, filtering, tracking. } \\
\text { Output type: N.A. }\end{array}$ & $\begin{array}{l}\text { Tasks: capture, segment, edge detection, edge tracking. } \\
\text { Output type: N.A. }\end{array}$ & $\mathrm{I}$ & A \\
\hline $14^{31}$ & $\begin{array}{l}\text { Tasks: capture, segment, filtering, mathematical } \\
\text { transform. } \\
\text { Output type: vector }\end{array}$ & $\begin{array}{l}\text { Tasks: capture, image division, edge detect, line extract } \\
\text { houghman, plot depth, segment, vertical pixel length, depth } \\
\text { curve analysis. } \\
\text { Output type: vector }\end{array}$ & III & $\mathrm{D}$ \\
\hline $15^{* 7}$ & $\begin{array}{l}\text { Tasks: capture, training, frame storage, subtract, segment, } \\
\text { filtering, feature extraction, classification. } \\
\text { Output type: vector }\end{array}$ & $\begin{array}{l}\text { Tasks: capture, training, frame storage, subtract, segment, } \\
\text { feature extraction, classification, morphology. } \\
\text { Output type: N.A. }\end{array}$ & $\mathrm{I}$ & $\mathrm{C}$ \\
\hline $16^{10}$ & $\begin{array}{l}\text { Tasks: capture, colour transform, segment, feature } \\
\text { extraction, classification. } \\
\text { Output type: N.A. }\end{array}$ & $\begin{array}{l}\text { Tasks: capture, colour transform, segment, } R O I, \mathrm{HOG} \\
\text { calculation, pose classification. } \\
\text { Output type: N.A. }\end{array}$ & II & B \\
\hline $17^{4}$ & $\begin{array}{l}\text { Tasks: capture, training, frame storage, subtract, segment, } \\
\text { feature extraction, tracking. } \\
\text { Output type: matrix. }\end{array}$ & $\begin{array}{l}\text { Tasks: capture, training, frame storage, subtract, segment, } \\
\text { object localization, boundary detection, tracking. } \\
\text { Output type: matrix. }\end{array}$ & II & B \\
\hline $18^{13}$ & $\begin{array}{l}\text { Tasks: capture, training, segment, filtering, feature } \\
\text { extraction, classification. } \\
\text { Output type: vector. }\end{array}$ & $\begin{array}{l}\text { Tasks: capture, training, downsample, skin colour classify, } \\
\text { low pass filtering, segment, feature extraction, gesture } \\
\text { classification. } \\
\text { Output type: vector. }\end{array}$ & II & $\mathrm{C}$ \\
\hline
\end{tabular}




\begin{tabular}{|c|c|c|c|c|}
\hline $19^{14}$ & $\begin{array}{l}\text { Tasks: capture, training, segment, filtering, classification. } \\
\text { Output type: vector. }\end{array}$ & $\begin{array}{l}\text { Tasks: capture, training, segment, filtering, body part } \\
\text { position, median filtering, skin colour classification. } \\
\text { Output type: vector. }\end{array}$ & I & $\bar{C}$ \\
\hline $20^{15}$ & $\begin{array}{l}\text { Tasks: capture, segment, filtering, feature extraction, } \\
\text { classification. } \\
\text { Output type: vector. }\end{array}$ & $\begin{array}{l}\text { Tasks: capture, } R O I \text {, edge detect, segment, feature } \\
\text { extraction, feature matching. } \\
\text { Output type: vector. }\end{array}$ & II & $\mathrm{C}$ \\
\hline
\end{tabular}

\section{REFERENCES}

1. S. Hengstler, "Stereo Vision in Smart Camera Networks," Stereo Vision, InTech Publisher, 73-90 (2008).

2. Rinner, B. Winkler, T., Schriebl, W., Quaritsch, M. and Wolf, W., "The evolution from single to pervasive smart cameras," ACM/IEEE Intl. Conf. on Distributed Smart Cameras, 1-10, (2008).

3. Teixeira, T., Lymberopoulos, D., Culurciello, E., Aloimonos, Y., and Savvides, A., "A Lightweight Camera Sensor Network Operating on Symbolic Information," 1st Workshop on Distributed Smart Cameras, (2006).

4. Kulkarni, P., Ganesan, D., Shenoy, P. and Q. Lu., "SensEye, A multi-tier camera sensor network," Intl Multimedia Conf. archive, $229-238$ (2005).

5. Hengstler, S., Prashanth, D., F. Sufen, H. Aghajan, "MeshEye: A Hybrid-Resolution Smart Camera Mote for Applications in Distributed Intelligent Surveillance," 6th Intl. Symposium on Information Processing in Sensor Networks, 360-369 (2007).

6. Bakkali, M., Carmona-Galá, R. and Rodriguez-Vazquez, A., "A prototype node for wireless vision sensor network applications development," 5th Intl Symp. on I/V Communications and Mobile Network, 1-4 (2010).

7. Orfy, A., El-Sayed, A. and ElHelw, M., "WASP: Wireless autonomous sensor prototype for Visual Sensor Networks," IFIP Wireless Days (WD), 1-5 (2010).

8. Xu, Z., Zhao, X. and Li, N., "Novel vehicle detection system based on line scan camera," 9th Intl. Conf. on Electronic Measurement \& Instruments, 1-748 (2009).

9. Se-Na, L., Bum-Jae, Y., Mee-Seub, L., Sang, O., Song-Soo, H. and Sang Heon, L., "Visual measurement of pile penetration and rebound movement using a high-speed line-scan camera," IEEE Intl. Conf. on Robotics and Automation, 4, 4307-4312 (2002).

10. Curran, P., Buckley, J., O’Flynn, B., Li, X., Zhou, J., Lacey, G. and O’Mathuna, S. C., "VAMP, A Vision Based Sensor Network for Health Care Hygiene," 14th nordic-baltic conf. on biomedical eng. and medical physics, 20 (2008).

11. Gasparini, L., Manduchi, R., Gottardi, M. and Petri, D., "An Ultralow-Power Wireless Camera Node: Development and Performance Analysis," IEEE Trans. on Instr. and Measurement, 60, 3824-3832 (2011).

12. Kerhet, A., Magno, M., Leonardi, F., Boni, A. and Benini, L., "A low-power wireless video sensor node for distributed object detection," journal of real-time image proc., 2, (2007).

13. Shi, Y., Taib, R. and Lichma, S., "GestureCam: A Smart Camera for Gesture Recognition and GestureControlled Web Navigation," Intl. Conf. on Contl, Automat., Robotics and Vision, 1-6 (2006).

14. Chen, W., Aghajan, H. and Kleihorst, R., "Real-Time Human Posture Reconstruction in Wireless Smart Camera Networks," Intl. Conf. on Information Proc. in Sensor Networks, 321-331 (2008).

15. Wolf, W., Ozer, B. and Lv, T., "Smart cameras as embedded systems," computer, 48-53 (2002).

16. Casares, M. and Velipasalar, S., "Adaptive Methodologies for Energy-Efficient Object Detection and Tracking With Battery-Powered Embedded Smart Cameras," IEEE Transactions on Circuits and Systems for Video Technology, 1438 - 1452 (2011).

17. Gonzalez, R. C. and Woods, R. E., "Digital Image Processing," Pearson Education, Inc. USA (2008).

18. Downes, I., Baghaei, L. and Aghajan, H.," Development of a Mote for Wireless Image Sensor Networks," in Proc. COGnitive systems with Interactive Sensors, (2006).

19. Imran, M., Khursheed, K., O’Nils, M. and Lawal, N., "Exploration of Target Architecture for a Wireless Camera Based Sensor Node," 28th Norchip Conf., 1-4 (2010).

20. Malik, A. W., Thörnberg, B., Cheng, X. and Lawal, N., "Real-time Component Labelling with Centre of Gravity Calculation on FPGA," Intl. Conf. on Systems, 23-28 (2011).

21. Walczyk, R., Armitage, A., and Binnie, T. D., "Comparative study on connected component labeling algorithms for embedded video processing systems," In IPCV'10. CSREA Press, 2 (2010). 
22. Hornberg, A., "Handbook of Machine Vision," Wiley-VCH Verlag GmbH \& Co, Germany (2006).

23. Yilmaz, A., Javed, O., and Shah, M., "Object tracking: A survey,” ACM Comput. Surveys, 38,1-45 (2006).

24. "IGOR Pro, version 4.0," WaveMetrics, Inc, 736-752, (2001). http://www.wavemetrics.net/.

25. Feddema, J. T., Lee, C. S. G. and Mitchell, O. R., "Weighted selection of image features for resolved rate visual feedback control," IEEE Trans. on Robotics and Automation, 31-47 (1991).

26. Pelissier, F. and Berry, F., "Design of a real-time embedded stereo smart cameras," Advanced concepts for intelligent vision systems, 6474 (2010).

27. CMOS sensor, MT9V032 (2001). Available : http://www.micron.com

28. Schriebl, W., Winkler, T., Starzacher, A., Rinner, B.," A pervasive smart camera network architecture applied for multi-camera object classification," Third ACM/IEEE Intl. Conf. on Distributed Smart Cameras, 1-8 (2009).

29. Rahimi, M., Baer, R., Iroezi, O. I., Garcia, J. C., Wrior, J., Estrin, D., Srivastava, M., "Cyclops: in situ image sensing and interpretation in wireless sensor networks," Proc. of the 3rd intl. conf. on Embedded networked sensor systems, 192-204 (2005).

30. Chen, P., Ahammad, P., Boyer, C., Huang, S., Lin, L., Lobaton, E., Meingast, M., Oh, S., Wang, S., Yan, P., Yang, A. Y., Yeo, C., Chang, L., Tygar, J. D., and Sastry, S. S., "CITRIC: A low-bandwidth wireless camera network platform," Second ACM/IEEE International Conference on Distributed Smart Cameras, 1-10 (2008).

31. Zhu, C. H., Hirahara, K. and Ikeuchi, K., "Street-parking vehicle detection using line scan camera," IEEE Proceeding Intelligent Vehicles Symposium, 575-580 (2003).

32. Dieber, B., Micheloni, C., and Rinner, B., "Resource-Aware Coverage and Task Assignment in Visual Sensor Networks," IEEE Trans. on Circuits and Systems for Video Technology, 1424 - 1437 (2011).

33. Hengstler, S., and Aghajan, H., "Application-oriented design of smart camera networks," 1st ACM/IEEE Int'l Conference on Distributed Smart Cameras, 12-19 (2007).

34. Tilak, S., Abu-Ghazaleh, N., and Heinzelman, W., "A Taxonomy of Wireless Micro-sensor Network Models," ACM SIGMOBILE Mobile Comp. Comm.. Review, 28 - 36 (2002).

35. Ferrigno, L., Marano, S., Paciello, V. and Pietrosanto, A., "Balancing computational and transmission power consumption in wireless image sensor networks," Poc. of the IEEE International Conference on Virtual Environments, Human-Computer Interfaces, and Measurement Systems, 61-66, (2005).

36. Fatemi, H., "Processor Architecture Design for Smart Cameras," $\mathrm{PhD}$ thesis, Technische Universiteit Eindhoven, (2007). 